\title{
Die Gutachter zum 65. Jahrgang (2014)
}

Die Herausgeber der Sozialen Welt bedanken sich bei folgenden Kolleginnen und Kollegen für ihre gutachterliche Unterstützung:

Ingrid Artus, Erlangen-Nürnberg

Katrin Auspurg, Konstanz

Ruth Ayass, Klagenfurt

Dirk Baier, Hannover

Roger Berger, Leipzig

Jürgen Beyer, Hamburg

Klaus Birkelbach, Essen

Christiane Bozoyan, München

Heinz Bude, Kassel

Gabriela Christmann, Erkner

Sérgio Costa, Berlin

Nina Degele, Freiburg

Claudia Diehl, Konstanz

Dietmar Fehr, Berlin

Christiane Funken, Berlin

Christian Ganser, München

Thomas Gautschi, Mannheim

Johannes Giesecke, Berlin

Matthias Groß, Leipzig

Daniela Grunow, Frankfurt

Jörg Haganah, Köln

Marcel Helbig, Berlin

Dieter Hermann, Heidelberg

Frank Hillebrandt, Hagen

Stefan Hirschauer, Mainz

Helmut Hirtenlehner, Linz

Stefanie Hiß, Jena

Roman Hoffmann, Wien

Marc Höglinger, Zürich

Boris Holzer, Bielefeld

Stefan Hornbostel, Berlin

Detlef Horster, Hannover

Monika Jungbauer-Gans, Erlangen-Nürnberg

Jochen Kade, Franfurt/Main

Bernhard Kittel, Wien

Thomas Koch, München

Klaus Kraemer, Graz

Reinhard Kreckel, Halle/Saale

Cornelia Kristen, Bamberg
Thomas Kron, Aachen

Ivar Krumpal, Leipzig

Volker Kruse, Bielefeld

Gesa Lindemann, Oldenburg

Daniel Lois, Neubiberg

Martina Löw, Berlin

Michael Mäs, Zürich

Heiner Minssen, Bochum

Lara Momesso, Tübingen

Julian Müller, München

Robert Neumann, Dresden

Natascha Nisic, Hamburg

Dietrich Oberwittler, Freiburg

Gunnar Otte, Mainz

Katrin Paula, Mannheim

Knut Petzold, Siegen

Birgit Pfau-Effinger, Hamburg

Angelika Poferl, Fulda

Manfred Prenzel, München

Ludger Pries, Bochum

Tilman Reitz, Jena

Christian Rieck, Frankfurt/Main

Jörg Rössel, Zürich

Katja Rost, Zürich

Merlin Schaeffer, Berlin

Michael Schillmeier, Exeter

Uwe Schimank, Bremen

Dominik Schrage, Lüneburg

Anna Spiegel, Bielefeld

Ursula Streckeisen, Bern

Barbara Sutter, München

Richard Traunmüller, Essex

Werner Vogd, Witten-Herdecke

Günther Voß, Chemnitz

Paul Windolf, Trier

Michael Windzio, Bremen

Tobias Wolbring, Zürich

Felix Wolter, Mainz

Eva-Maria Ziege, Bayreuth 